Gerión. Revista de Historia Antigua

ISSN: 0213-0181

http://dx.doi.org/10.5209/geri.71947

\title{
Inscripciones latinas inéditas de Casa del Olivo $^{1}$
}

\author{
Estela García Fernández²; María del Rosario Hernando Sobrino ${ }^{3}$
}

Recibido: 3 de mayo de 2020 / Aceptado: 21 de junio de 2020

Resumen. Presentamos aquí tres inscripciones romanas, inéditas y presumiblemente procedentes del territorio de la actual provincia de Córdoba, dos de ellas registradas en el segundo volumen de la autobiografía de Carlos Castilla del Pino titulada Casa del Olivo. Las dos primeras son de carácter funerario y, al menos la segunda, se acomoda en tipología y contenido a los parámetros usuales de la epigrafía funeraria de la antigua Colonia Patricia Corduba; por el contrario, la tercera, fragmentada, corresponde a un epígrafe de carácter métrico.

Palabras clave: epigrafía romana; epígrafes funerarios; inscripciones métricas; Baetica.

\section{[en] Unpublished Latin Inscriptions from Casa del Olivo}

Abstract. This paper shows and analyses three roman unpublished inscriptions. They come, probably, from the territory of the Córdoba province and two of them were registered by Carlos Castilla del Pino in the second volume of his autobiography, entitled Casa del Olivo. The first two are of a funerary nature, and in particular the typology and content of the second fits with, the usual parameters of the funerary epigraphy of the ancient Colonia Patricia Corduba. The third, a fragmentary inscription, corresponds to a metric one.

Keywords: Roman Inscriptions; Funerary Inscriptions; Metric Inscriptions; Baetica.

Sumario: 1. Introducción. 2. Inscripción funeraria de Numisia Laberiana. 3. Inscripción funeraria de Clodia Primitiva. 4. Inscripción métrica en estado fragmentario. 5. Conclusiones. 6. Referencias bibliográficas.

Cómo citar: García Fernández, E.; Hernando Sobrino, Mª R. (2020): Inscripciones latinas inéditas de Casa del Olivo, en Gerión 38/2, 549-562.

1 Este trabajo se enmarca en los proyectos de investigación Redes AVIPES-CM (ref. H2019-HUM/5742), de la Comunidad de Madrid/Fondo Social Europeo, Nuevas bases documentales para el estudio histórico de la Hispania romana en época republicana: onomástica y latinidad (III-I a.C.) (ref. HAR2015-66463-P), del Ministerio de Economía y Competitividad, y Corpus de Manuscritos de la Biblioteca Nacional de Madrid para la Historia Antigua de España (ref. PID2019-109530GB), del Ministerio de Ciencia, Innovación y Universidades, así como en los Grupos de investigación de la UCM Ciudades Romanas (ref. 930692) y TEAPIMeG (ref. 930750). Agradecemos a los evaluadores anónimos sus sugerencias, que han contribuido a mejorar la versión definitiva de este trabajo.

2 Universidad Complutense de Madrid.

E-mail: estgarci@ucm.es

ORCID: 0000-0002-4486-7859

3 Universidad Complutense de Madrid - Archivo Epigráfico de Hispania.

E-mail: mrhernando@ghis.ucm.es

ORCID: 0000-0002-7509-7877 


\section{Introducción}

Por lo común, y como bien puede deducirse de algunos de los artículos que forman parte de este mismo volumen, la detección de inscripciones romanas inéditas "en papel" se produce al hilo del cotejo y análisis de la documentación manuscrita conservada en bibliotecas y archivos. Sin embargo, no ha sido éste el caso de los epígrafes que aquí presentamos.

Las inscripciones -en realidad dos de ellas, como más adelante se explicaráfueron detectadas en un contexto más próximo al otium que a la labor investigadora, cuando una de nosotras abordó la lectura del segundo volumen ${ }^{4}$ de la autobiografía del neurólogo, psiquiatra y escritor Carlos Castilla del Pino (San Roque, Cádiz 1922 - Castro del Río, Córdoba 2009), titulada Casa del Olivo. ${ }^{5}$

Si bien es conocido de modo fundamental por sus sobresalientes méritos profesionales en el ámbito de la psiquiatría y su militancia política, ${ }^{6}$ debe destacarse que Castilla del Pino fue igualmente una figura relevante en el ámbito de las Letras; de hecho, su innegable talento como escritor ${ }^{7}$ le franqueó las puertas de la Real Academia de la Lengua, en la que ocupó el sillón «Q» desde el 7 de marzo de 2004, fecha de su discurso de toma de posesión o recepción pública, hasta su fallecimiento. Por la misma razón, se hizo acreedor de numerosos galardones y distinciones, tales como el Premio María Zambrano (2000), la Medalla de Oro del Círculo de Bellas Artes de Madrid (2002) y la Medalla de Oro de la Universidad Internacional Menéndez Pelayo (2002); asimismo fue nombrado Hijo Predilecto de Andalucía e Hijo Predilecto de San Roque en 1985 y, en 2002, Hijo Predilecto de Cádiz.

Hombre de gran cultura, el gaditano se muestra en su autobiografía no sólo como un testigo de excepción de la España del franquismo, sino también como un defensor, sensible y concienciado, del patrimonio histórico-arqueológico. Las páginas de su autobiografía abundan, por ello, en referencias a los materiales y hallazgos arqueológicos de su entorno, unos y otros de diferentes épocas y de entidad diversa, no faltando entre ellos los epígrafes de época romana.

Son, en concreto, dos las referencias a inscripciones hispanorromanas que podemos encontrar en la Casa del Olivo; en ambos casos, Castilla del Pino apenas si señala más que su existencia pero, por fortuna, menciona los antropónimos que se pueden leer en las mismas y, al citar una de ellas (véase infra, § 3 ) indica, además, que forma parte de los objetos con que su esposa y él han construido "su" espacio en la casa que da nombre a la autobiografía, situada en Castro del Río (Córdoba).

Una vez comprobado que, en atención a los antropónimos registrados, dichas inscripciones parecían inéditas, decidimos contactar con la Dra. Celia Fernández

\footnotetext{
El primero vio la luz con el título Pretérito imperfecto. Autobiografia (1922-1949), Barcelona, Tusquets, 1997. Como se especifica en la bibliografía final, se leyó la edición de Tusquets publicada en el año 2004; la obra fue tomada en préstamo de la Biblioteca Municipal Eugenio Trías. Casa de Fieras de El Retiro (Paseo Fernán Núñez 24, 28009 Madrid), en la que está catalogada con la signatura B CAS [https://gestiona3.madrid.org/ biblio_publicas/cgi-bin/abnetopac/O9339/ID78adf135/NT10].

6 Estuvo afiliado al Partido Comunista hasta 1980, militancia que explica se le negase, en repetidas ocasiones, el acceso a la cátedra de Psiquiatría, que no obtendría hasta la llegada del PSOE al poder en 1983.

7 Colaborador habitual de El País, fue autor de diferentes ensayos y monografías sobre psiquiatría, así como de las novelas Discurso de Onofre (Ediciones Península, Barcelona, 1977) y Una alacena tapiada (Ediciones Península, Barcelona, 1991).
} 
Prieto, ${ }^{8}$ viuda de Castilla del Pino, con la intención de verificar su existencia. Gracias a su amable colaboración, que desde aquí queremos agradecer de modo expreso, podemos hoy presentar estas líneas.

De acuerdo con la información proporcionada por la Dra. Fernández Prieto, ${ }^{9}$ de las dos inscripciones citadas en la Casa del Olivo sólo una permanece en el lugar (aquí § 3); lamentablemente, no se ha localizado la segunda (véase § 2), pero sí una tercera (infra, § 4) que no había sido registrada en la autobiografía.

En ningún caso conocemos la procedencia exacta de las piezas, pero todo hace sospechar -y en algún caso así parecen permitirlo los escuetos datos proporcionados por Castilla del Pino- que se trata de piezas "locales", esto es, del propio territorio cordobés.

\section{Inscripción funeraria de Numisia Laberiana}

Comenzamos, precisamente, con la inscripción que no se ha podido localizar; Castilla del Pino hace referencia a ella al describir el entorno de la "Casa del Olivo":

Nos bañábamos en pozas del arroyo del Duende, próximo a El Mochuelo. A unos quinientos metros observamos una canalización árabe, que pudimos seguir más de un kilómetro, y que aún llevaba agua, la que en Córdoba se ha conocido como «agua del cabildo», y de la que todavía se surten, o se surtían, algunas casas y fuentes. Un día, unos obreros que trabajaban en las proximidades me obsequiaron con una lápida funeraria de una joven romana (Numisia Laberiana, Anno $X X)$ y poco después me llamaron de nuevo: habían encontrado varias tumbas árabes con tejas de cerámica (conservo los restos óseos que recogí en una de ellas)... ${ }^{10}$

Y hasta aquí los datos. En ausencia del original, todo lo más que podemos indicar es que parece posible presumir, dadas las circunstancias en que se produjo su entrega, que la inscripción procediese del entorno inmediato, quizá del territorio de la antigua comunidad de Soricaria,${ }^{11}$ que formó parte del conventus Astigitanus.

Resulta imposible determinar si en el epígrafe constaban otros datos que, al menos en determinados periodos, fueron usuales en la epigrafía funeraria: la consagración a los Manes, la presencia de fórmulas funerarias o, también, de dedicantes o epítetos dedicados a la difunta. Porque obviamente, y como bien indica el autor, se trataba de una inscripción funeraria, como la secuencia ann(orum) $X X$ evidencia.

\footnotetext{
Profesora Titular de Teoría de la Literatura y Literatura Comparada de la Facultad de Filosofía y Letras de la Universidad de Córdoba.

9 Quien tuvo, además, la gentileza de proporcionarnos las fotografías y realizar todas las mediciones necesarias para la edición completa de los epígrafes que aquí se presentan.

10 Castilla del Pino 2004, 286.

11 La identificación del solar concreto de esta comunidad, mencionada únicamente por el Bellum Hispaniense (24 y 27) en el marco del enfrentamiento entre Pompeyo y César (45 a.C.), sigue sujeta a discusión, si bien no faltan quienes la sitúan en el yacimiento del "Cerro de las Cuevas de Sequeira", que se reparte entre los términos municipales de Castro del Río y Nueva Carteya (cf. Morena López 1998; Melchor Gil 2005, 370-372). Para su integración en el conventus Astigitanus véanse, entre otros, Campos et alii 2018, 306-307, con una síntesis sobre los problemas relativos a su identificación.
} 
No hay más objeto de análisis, en consecuencia, que el nombre de la joven de cuya memoria se hacía eco el epígrafe: Numisia Laberiana. El nomen Numisius, $-a^{12}$ no es desconocido - sin ser particularmente abundante- en el registro epigráfico hispano, tal y como se deduce de una primera comprobación en el útil repertorio del profesor Abascal. ${ }^{13}$ En éste se advierte que la mayor parte de los testimonios censados se localizan en la Citerior -especialmente en Carthago Nova, Tarraco y Barcino-, resultando muy escasa su presencia en la Bética, con apenas siete testimonios, ${ }^{14} \mathrm{y}$ en Lusitania, con sólo cuatro; sin embargo, con los años, y especialmente con la publicación de los fascículos de $C I L \mathrm{II}^{2}$ correspondientes a los conventus Astigitanus $\left(C I L \mathrm{II}^{2} / 5\right)$ y Cordubensis $\left(C I L \mathrm{II}^{2} / 7\right)$, el registro bético se ha visto notablemente incrementado, alcanzando ya los quince registros. ${ }^{15}$ Además de su importancia en términos estadísticos, destaca de este aumento el hecho de que muestra una considerable concentración de Numisii precisamente en las tierras cordobesas, en las que se registran seis de los quince testimonios béticos $;{ }^{16}$ de hecho, los especialistas consideran, valorando especialmente para ello su presencia epigráfica en el teatro romano de la propia Colonia Patricia, ${ }^{17}$ que los Numisii se encontraban "entre las familias más conspicuas"18 de la capital provincial. A los testimonios relativos a esta familia vendría a sumarse, por tanto, el epígrafe que aquí presentamos.

Si el nomen Numisius, - $a$, se encuentra relativamente bien documentado, no podemos decir lo mismo del cognomen que porta la difunta: Laberiana ${ }^{19}$ Si nuestros datos son correctos, el testimonio cordobés constituiría el segundo documentado en Hispania y el primero en femenino pues, hasta la fecha, el único individuo atestiguado con este nombre no era sino el Conimbrigensis M. Aurelius Laberianus. ${ }^{20}$ Del mismo modo, cabe destacar que este cognomen es igualmente escaso en la epigrafía del mundo romano en su conjunto; una simple búsqueda en la bases de datos de amplio espectro basta para comprobarlo: sólo diez testimonios, contando entre ellos el hispano que acabamos de mencionar; un registro decididamente parco que sólo en tres ocasiones rebasa el marco de la península italiana, en la que se concentra de modo evidente. ${ }^{21}$ Así pues, y pese a su aparente banalidad, el epígrafe reviste cierto

\footnotetext{
Solin - Salomies 1988, 129.

Abascal Palazón 1994, 191-192.

4 Y bastante repartidos: dos en Italica (CIL II 1154 y EE IX, 191), uno en Urso (CIL II 5447), uno en Corduba $\left(C I L\right.$ II $2298=C I L$ II $\left.^{2} / 7,503\right)$, uno en Nueva Carteya (CIL II $\left.1599=C I L I^{2} / 5,354\right)$, uno en Regina $(E E$ IX, 185 $=$ CIL II $2 / 7,984)$ y uno en Gades (CIL II 1856).

15 La detección de los registros relativos a la presencia del nomen Numisius en Hispania se ha completado con la consulta de las bases de datos de Clauss-Slaby (EDCS) e Hispania Epigraphica Online (HEpOL) [consultadas el día 15 de septiembre de 2018].

16 Súmense, a los dos citados en la nota previa (n. 14), de Corduba y Regina, los epígrafes correspondientes a CIL $\mathrm{II}^{2} / 7,251$; CIL II $/ 7$, 326; CIL II ${ }^{2} / 7$, 608a y Ventura Villanueva 2002, 266-269 (de donde HEp 13, 2003/2004, 270).

17 Además del ya citado epígrafe correspondiente a $H E p$ 13, 2003/2004, 270, que conmemora la erección de una estatua a L. Numisius L. f. Se[rg.] Sollers en el teatro, sabemos que uno o varios de sus miembros tenían reservados asientos en este edificio (cf. Ventura Villanueva 2002, 265-266).

18 Vaquerizo Gil 2018, 81; en el mismo sentido Ventura Villanueva 1999, 72; 2002; 2009, 389, Pérez Zurita 2017, 145, y Rodríguez Neila 2017, 388, por sólo citar algunos trabajos relevantes.

19 Cognomen derivado del nomen Laberius, cf. Kajanto 1965, 148; Solin - Salomies 1988, 348.

20 CIL II 370 (Conimbriga); Abascal Palazón 1994, 394; Navarro Caballero - Ramírez Sádaba (coords.), 2005, 209 (en adelante Grupo Mérida). Véase, también, su registro en ADOPIA Lusitania [http://adopia.huma-num.fr/ es/; base consultada el día 22 de junio de 2020], donde, frente a la relativa abundancia del nomen Laberius, el epígrafe portugués sigue constando como único testimonio del cognomen Laberianus.

21 Tres epígrafes proceden de la propia Roma (CIL VI 2405, CIL VI 37310 y CIL VI 37312), uno de Ostia, en la
} 
interés para el ámbito de los estudios antroponímicos.

En ausencia de la pieza, que esperamos pueda ser recuperada, cualquier ensayo de datación no puede ser sino hipotético. No obstante, si el texto efectivamente se limitase a la lectura proporcionada por Castilla del Pino, habría que otorgarle una fecha temprana, posiblemente la primera mitad del s. I d.C.

\section{Inscripción funeraria de Clodia Primitiva}

Mencionada por Castilla del Pino simplemente como "lápida de Clodia", ${ }^{22}$ la inscripción corresponde a una placa que creemos elaborada en mármol y que -si se exceptúa la fractura de su ángulo inferior derecho y la pérdida parcial de la misma esquinaparece estar completa (Fig. 1).

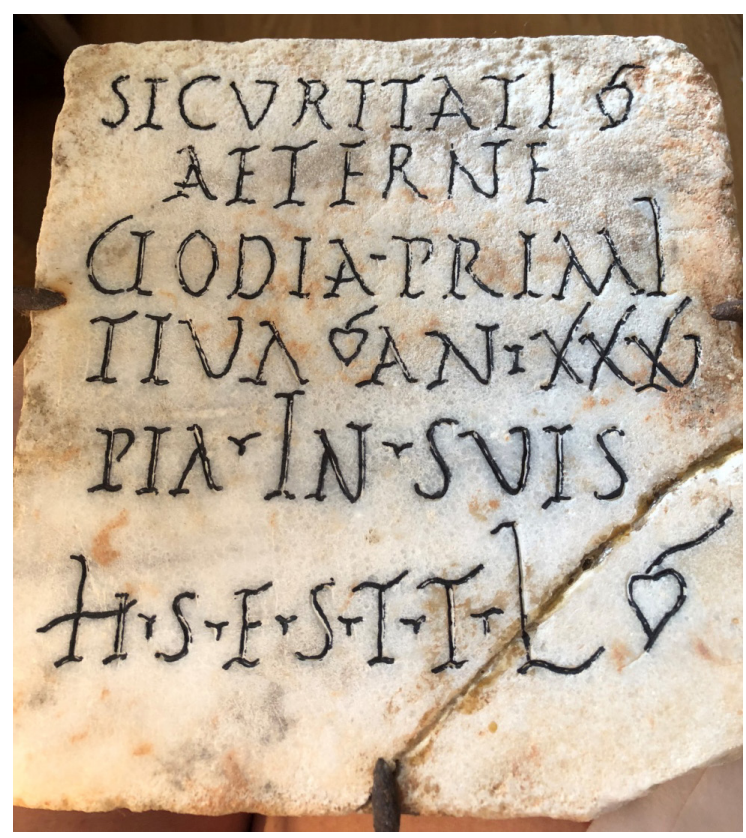

Fig. 1. Inscripción funeraria de Clodia Primitiva ( Celia Fernández Prieto).

Sus medidas son: $21 \times 19,5 \times 2,8-2 .{ }^{23}$ De acuerdo con estas dimensiones, estaríamos ante una "placa de pequeño formato" ${ }^{24}$ un tipo de soporte muy usual en

Regio I (CIL XIV 692), otro de Canusium, en la Regio II (CIL IX 338), y otro más de Patavium, en la Regio X (CIL V 3000). Los restantes testimonios se reparten entre Africa proconsularis (CIL VIII 24019, de Uthina), Pannonia Inferior (CIL XVI 136, de Aquincum) y Lusitania, con el ya citado epígrafe de Conimbriga. Los datos han sido tomados de la base de datos de Clauss-Slaby (EDCS) [consultada el día 16 de septiembre de 2018].

22 Castilla del Pino 2004, 458.

23 Expresamos aquí todas las medidas en centímetros.

24 Para distinguirlas de las que, para los conventus Cordubensis y Astigitanus, se denominan de "gran formato", se tiene en cuenta la anchura, especialmente, y el grosor de la pieza: se considera que pertenecen al grupo de "placas de pequeño formato" las que presentan una anchura menor a los $50 \mathrm{~cm}$ y un grosor inferior a $10 \mathrm{c} 10 \mathrm{~cm}$ (Ruiz Osuna 2009, 28 y 102, n. 75). Para Ventura, sin embargo, el grosor de estas placas debe ser inferior a los 
el conjunto epigráfico cordobés (al que, como ya avanzamos, bien pudiera pertenecer este testimonio), en el que se documentan más de un centenar de ejemplares. Se trata siempre de placas ejecutadas en mármol y que, como la que aquí nos ocupa, carecen de molduras. ${ }^{25}$ Constituyen el soporte de mayor éxito desde el s. II d.C. y hasta la primera mitad del s. III d.C., siendo especialmente empleado por los sectores más humildes de la población, tanto por ingenuos como por libertos; se solían encastrar en otros monumentos funerarios (construcciones de obra, loculi de columbarios, sillares, cipos, cupae, altares, estelas, etc., por lo común realizados en arenisca local) o en el mismo suelo. ${ }^{26}$

Por lo que respecta a sus características paleográficas, y hasta donde nos permite llegar el repaso con tinta negra a que han sido sometidas las grafías, el texto está inscrito en letras librarias de regular factura; sus medidas medias son: 1,8-1,5; debiendo destacarse las siguientes grafías: I longa de lín. 3: 2,7; I longa de lín. 5: 2,5; L de lín. 6: 3,2; del mismo modo creemos, aunque no lo apreciamos inicialmente, que la I final de lín. 1 es igualmente longa. ${ }^{27} \mathrm{Se}$ advierte un nexo XV en la lín. 4.

El texto fue distribuido en el soporte con bastante cuidado: centrado y bien paginado en líneas generales. Del mismo modo, se encuentra correctamente puntuado, haciendo uso de hederae y de interpunciones que responden al tipo denominado "trisquel"; ambas formas se alternan con una aparente intencionalidad estética: las hojas de hiedra parecen emplearse de un modo un tanto decorativo, apareciendo al final de la primera y última línea y en el centro de la línea intermedia (lín. 4).

Su lectura es la siguiente:

$$
\begin{gathered}
\text { Securitati Chedera } \supset \\
\text { aetern }(\text { a)e } \\
\text { Clodia } \cdot \text { Primi- } \\
\text { tiva } C \text { hedera } \supset \text { an }(\text { norum }) \cdot X X \hat{X} V \\
\text { pia } \cdot \text { in } \cdot \text { suis } \\
h(\text { ic }) \cdot s(\text { ita }) \cdot e(\text { st }) \cdot s(\text { it }) \cdot t(\text { ibi }) \cdot t(\text { erra }) \cdot l(\text { evis }) \text { Chedera } \supset^{28}
\end{gathered}
$$

Resulta de gran interés la expresión que abre el texto, la referencia a securitas aeterna, pues no sólo resulta muy extraña en la epigrafía peninsular, sino que cuenta con escasísimos paralelos exactos a escala imperial. ${ }^{29}$ En el conjunto epigráfico de Hispania sólo se documenta en la propia Corduba y, en ésta, sólo en

$5 \mathrm{~cm}$, parámetro al que también se ajusta el ejemplar que aquí presentamos (Ventura 2001, 175).

25 Ventura 2001, 175.

26 Véase Ventura 2001, 175; Ruiz Osuna 2007, 46; Vaquerizo Gil 2008, 97; 2010, 136. Más información sobre el mundo funerario de la Corduba romana, además de los títulos ya citados, en Vaquerizo Gil - Garriguet (coords.) 2001; Vaquerizo Gil 2002; 2010, en especial 104-142; Ruiz Osuna 2007; 2010, por sólo citar los más relevantes.

27 Para el uso de la I longa en la epigrafía de los conventus Astigitanus y Cordubensis véase Martín Adán 2015, en especial $45-57$ y $112-137$.

28 En la edición de las inscripciones seguimos los signos diacríticos empleados en la revista Hispania Epigraphica.

29 Para un completo análisis de la aparición del término securitas en la epigrafía véase el trabajo de Haensch 2016; contabiliza este autor 240 inscripciones, de las que 165 corresponden a epitafios: "avec trois grands types de formules (et des sous-types). Ou bien l'on se réfère seulement à la securitas (environ 35 témoignages), ou l'on s'adresse aux Dii Manes et à la securitas perpetua (environ 105 témoignages) ou bien encore aux Dii Manes et à la securitas aeterna (plus de 20 témoignages)"; tales tipos, precisa, resultan del desarrollo de hábitos epigráficos regionales y no de una evolución cronológica o de la pertenencia a un determinado medio social (Haensch 2016, 35 , con tablas de registros en 36-44). 
dos epígrafes, en uno de los cuales sigue a la consagración a los dioses Manes. ${ }^{30}$ Por lo que hace a su consignación en otras áreas del Imperio, una búsqueda en las bases de datos basta para confirmar la escasez de su registro, tanto en la forma en que aquí se constata como con los términos en posición invertida (aeterna securitas), y ya se registre aislada o integrada en fórmulas más amplias, como Dis Manibus et securitati aeternae o Dis Manibus et securitati aeternae et memoriae o et memoriae quieti, entre otras variantes. ${ }^{31}$ Así, y además de los cordobeses, hemos contabilizado apenas 20 registros, que se muestran bastante repartidos por el territorio imperial, ${ }^{32}$ si bien parecen concentrarse de modo significativo, como bien señala Haensch, ${ }^{33}$ en las provincias galas en su conjunto, con dos testimonios en la Gallia Narbonensis ${ }^{34}$ y siete en la Lugdunensis, todos ellos procedentes de Lugdunum. ${ }^{35}$

Ya Haensch señala que, en numerosas ocasiones, es muy difícil determinar si se debe considerar securitas como una deidad o como un concepto abstracto; no obstante, nos sumamos aquí a su opinión de que en las inscripciones funerarias se emplea frecuentemente, y al menos en el caso de los epígrafes del s. III d.C., pensando en la protección de la tumba, de ahí el uso de la minúscula. ${ }^{36}$

Frente a la singularidad de la fórmula de apertura, el formulario final responde a estereotipos bien conocidos, aunque la expresión pia in suis suele aparecer preferentemente abreviada, no por extenso, como lo hace en esta inscripción.

El nombre de la difunta se lee con claridad $y$, de no considerar que se ha omitido la grafía final de la marca de caso, -ae, se registra en nominativo, como sucede en el ya citado paralelo cordobés correspondiente a $C I L \mathrm{II}^{2} / 7,418 ;{ }^{37}$ está compuesto por el nomen Clodia y el cognomen Primitiva. Los Clodii ${ }^{38}$ están muy bien documentados en el registro epigráfico hispano, ${ }^{39}$ mostrando una notable dispersión en sus testimonios; no obstante, parecen afectar una cierta concentración en el territorio de la Baetica (sin faltar ejemplos en la propia Corduba ${ }^{40}$ y en las diversas comunidades integradas en el conventus del que fue capital) ${ }^{41}$ y en los

30 CIL II ${ }^{2} / 7,418$ : D(is) M(anibus) s(acrum) / aeternae securita ${ }^{\beta}$ ti Auge an(norum) XXXV / p(ia) i(n) s(uis) $h($ ic) $s$ (ita) e(st) s(it) t(ibi) t(erra) l(evis); correspondiente, también, a una "placa de pequeño formato". CIL $\mathrm{II}^{2} / 7$, 508: Securitati aeternae / Paciae Saturninae piissimae indulgen ${ }^{\beta}$ tissimae omnium suorum cum qua vixi ann(os) XXXII / et bilem cum ea numquam habui Iul(ius) Baeticus mar(itus) / eius et Pacia Natalis lib(erta) fecer(unt) excessit ann(is) XXXXVIII $/ 0$ p(ia) in s(uis) h(ic) s(ita) e(st) s(it) t(ibi) t(erra) levis.

31 Búsqueda realizada en la base de datos EDCS [consultada el día 19 de septiembre de 2018], completada con el citado trabajo de Haensch 2016.

32 Encontramos esta fórmula en epígrafes funerarios de Roma (AE 1997, 152), en Ostia (CIL XIV 949; Latium et Campania, Regio I), Bonna (AE 1978, 570; Germania Inferior); Solva, mansio (RIU 3, 792; Pannonia Superior), Lambaesis (CIL VIII 3763, Numidia), Thamugadi (AE 2003, 2018; Numidia), Dolyche (AE 2011, 1467, Syria).

33 Haensch 2016, 35

34 CIL XII 409 (Massilia) y CIL XII 1971 (Vienna).

35 CIL XIII 1958, CIL XIII 1962, CIL XIII 2094, CIL XIII 2120, CIL XIII 2169, ILTG 225 y AE 1973, 335.

36 Haensch 2016, 35, n. 38.

37 Véase supra, n. 30. Lo usual, en efecto, es que la fórmula securitati aeternae vaya seguida del nombre del difunto en genitivo.

38 Solin - Salomies 1988, 57.

39 Abascal Palazón 1994, 113-114.

40 Así: $C I L \mathrm{II}^{2} / 7,235, C I L \mathrm{II}^{2} / 7,281, C I L \mathrm{II}^{2} / 7,292$, CIL II $2 / 7,304, C I L \mathrm{II}^{2} / 7,332$ y $C I L \mathrm{II}^{2} / 7,439$.

41 Por ejemplo: $C I L \mathrm{II}^{2} / 7,160$ (de Epora) y $C I L \mathrm{II}^{2} / 7$, 756 (de Solia). 
conventus levantinos de la Citerior, ${ }^{42}$ siendo menos significativa su presencia en la Lusitania. ${ }^{43}$

El cognomen Primitivus, $-a$, por su parte, resulta bien conocido, ${ }^{44}$ aunque el censo elaborado por Abascal no supera los 25 registros para toda Hispania y, en el mismo, es notoria la casi total ausencia de este nombre del conjunto epigráfico bético; ${ }^{45} \mathrm{sin}$ embargo, no faltan ahora testimonios en este territorio, si bien es cierto que en el conventus Cordubensis sigue siendo decididamente escaso. ${ }^{46}$

La tipología del soporte, los rasgos paleográficos y las fórmulas empleadas permiten datar el texto a finales del s. II d.C. o inicios del s. III d.C.

\section{Inscripción métrica en estado fragmentario}

Nos encontramos, en este caso, ante un fragmento que pudiera corresponder a la parte izquierda de una inscripción presumiblemente ejecutada en mármol; se encuentra rota por todos sus márgenes, sin embargo, dado el amplio espacio en blanco perceptible al inicio de cada línea de escritura, es posible estimar que no ha perdido texto por su lateral izquierdo (Fig. 2).

Sus medidas actuales son: $(11,5) \times(17,5) \times 4$; en atención al grosor marcado, parece lícito considerar que nos encontramos ante otra placa, sin que podamos, por razones obvias, ni precisar si fue de gran o de pequeño formato ni saber si presentaba o carecía de molduras. ${ }^{47}$

Por lo que se refiere a sus características paleográficas, y si el repaso de los rasgos con tinta negra no nos despista a este respecto, nos encontramos ante capitales cuadradas con tendencia a la libraria (véase, en particular, el trazado de la letra $\mathrm{T}$ en lín. 3), que presentan remates triangulares muy próximos a la cola de golondrina (especialmente visibles en todas las $\mathrm{S}$ del epígrafe, en la $\mathrm{N}$ de lín. 3 y en el extremo inferior izquierdo de la $\mathrm{R}$ de lín. 4, grafías cuyos ápices no fueron alcanzados por la tinta negra). Su tamaño es muy regular, sujetándose a un módulo de 1,5 cm que sólo parece romperse -siempre según la fotografía- en el caso de la primera $\mathrm{V}$ de lín. 4, ligeramente más pequeña.

Aparentemente, el texto se encuentra bien paginado, destacando de manera muy particular en su composición el acusado sangrado en la lín. 2. Del mismo modo, parece encontrarse correctamente puntuado, siendo perceptibles en todas las líneas interpunciones triangulares cuyo vértice se orienta de modo mayoritario hacia abajo y hacia la derecha; sólo la interpunción de lín. 3, de no engañarnos el ángulo de la fotografía, presenta el triángulo con el vértice hacia abajo. ${ }^{48}$

42 De hecho, en Játiva se registra una fémina que porta exactamente el mismo nombre: Clod(ia) Primitiva (CIL II $3630=$ Corell 1994, esto es, IRST 17).

43 Grupo Mérida 2003, 147.

44 Cf. Kajanto 1965, 74-75 y 290; Solin - Salomies 1988, 383.

45 Abascal Palazón 1994, 466.

46 Sólo tres epígrafes lo registran: $C I L \mathrm{II}^{2} / 7,61$ y $C I L \mathrm{II}^{2} / 7,64$, ambos de $I$ sturgi, y $C I L \mathrm{II}^{2} / 7$, 739, de Detumo, correspondiendo este último testimonio al nombre único de un esclavo.

47 Las placas de gran formato se caracterizan, básicamente, por presentar una anchura superior a los $50 \mathrm{~cm}$, con una altura entre 30 y $60 \mathrm{~cm}$ y un grosor similar al ya señalado para las de pequeño formato: inferior a $10 \mathrm{~cm}$; conformarían piezas de silueta cuadrangular con tendencia a la horizontalidad (Ruiz Osuna 2009, 28 y 95, n. 63).

48 Sobre las interpunciones triangulares y su significación cronológica en la epigrafía funeraria de la Bética, véase 


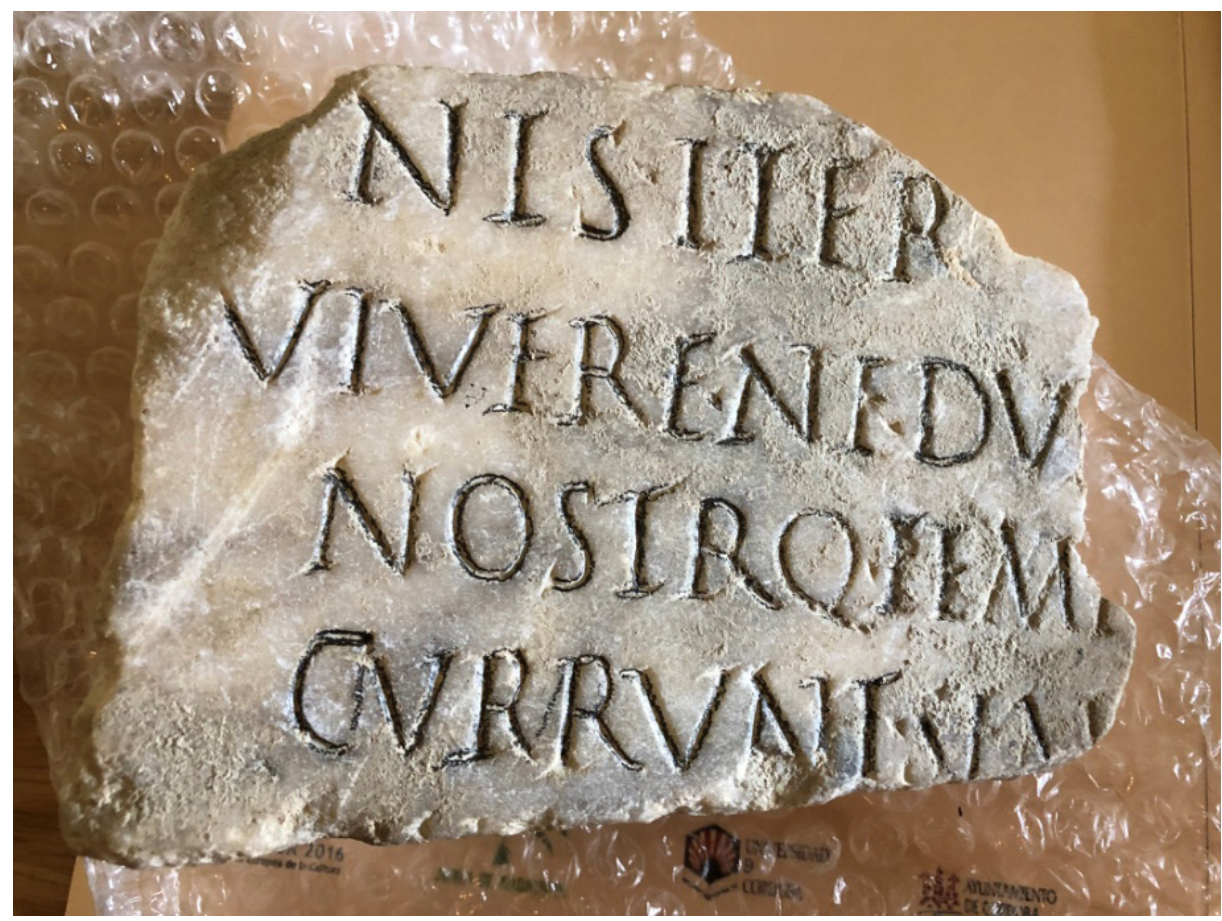

Fig. 2. Fragmento de inscripción (C) Celia Fernández Prieto).

La lectura del texto no comporta, en sentido estricto, demasiados problemas, salvo en el lateral derecho, donde las concreciones, el repaso con tinta de las letras y el desprendimiento de una lasca justo al final de la primera línea conservada generan algunas dudas.

Nuestra lectura es la que sigue:

$$
\begin{gathered}
\text { NIS } \cdot \text { ITER [- - ] } \\
\text { VIVERE } \cdot \text { NE } \cdot \text { DV [- - ] } \\
\text { NOSTRQ } \cdot \text { TEM }+[- \text { - - } \\
\text { CVRRVNT } \cdot \text { SIM }[---]
\end{gathered}
$$

El resto perceptible al final de lín. 3 corresponde a un trazo vertical que, en principio, tanto puede pertenecer a una I como a una $\mathrm{P}$ o una $\mathrm{T}$.

A primera vista resulta obvio que no nos encontramos ante un texto de contenido ordinario y, ello, a pesar de que apenas si se puede confirmar la presencia de dos términos que parecen estar completos. Sin embargo, estos términos, conjugados con la estructura aparente del texto, nos hicieron considerar de modo prácticamente inmediato la posibilidad de que nos encontrásemos ante un epígrafe de carácter métrico. 
Como bien nos indicó la Dra. Isabel Velázquez, a quien agradecemos la ayuda prestada, resultan particularmente significativas a este respecto dos líneas: la lín. 2 , donde los restos conservados -vivere ne $d u[---]-$ se acomodan a un esquema dactílico, y la lín. 3, en la que, asumiendo que en el primer término se ha omitido una vocal y que, en el segundo, el resto conservado bien podría corresponder a una $\mathrm{P}$, cabría entender nostr $<a>$ que temp [ora], o similar, de donde resultarían también dos dáctilos.

Menos evidentes resultan las lín. 1 y 4 . Por lo que respecta a la primera, parece obvio que los primeros rasgos conservados (-nis) corresponden a una palabra que se iniciaba en la línea precedente, aspecto éste que, en principio, podría dificultar su consideración desde el punto de vista métrico. Por lo que se refiere a la segunda, tampoco podemos asegurar que no suceda lo mismo, pues currunt bien puede constituir la continuación de una forma compuesta como, por ejemplo, concurrunt. ${ }^{49}$

Llegados a este punto, no hubiésemos podido avanzar en la comprensión de la inscripción de no mediar la inestimable cooperación de los Dres. Rocío Carande y Alberto Bolaños, cuya solvencia en materia de epigrafía métrica es de todos conocida: ${ }^{50}$ vaya desde aquí nuestra gratitud para ambos. Las líneas que siguen son, de hecho, la exposición de sus datos y sugerencias, que hacemos nuestros.

En primer lugar, señalan que, a la vista del texto-completo por su lateral izquierdo, como ya se apuntó-, resulta claro que no hay coincidencia verso-línea y que es muy posible que hubiese un verso cada dos líneas. Del mismo modo, advierten que el sangrado, aquí evidente en la lín. 2 del epígrafe, indica un tipo de compaginación que se suele utilizar precisamente en estos casos, tal y como puede advertirse en, por ejemplo, el carmen correspondiente a CIL VI 7898 y, también -dato que resulta de gran interés- en $C I L \mathrm{II}^{2} / 5,506$, de Montemayor (Fig. 3), localidad que dista apenas $28 \mathrm{~km}$ de Castro del Río, de modo que se podría pensar en una suerte de paginación local.

Partiendo de esta premisa, nos proporcionan el siguiente análisis:

a) Línea 1. Considerando la pérdida de texto al final de la línea, tanto por el desprendimiento de una lasca como por la rotura general de la pieza en el lateral derecho, se podrían proponer dos alternativas:

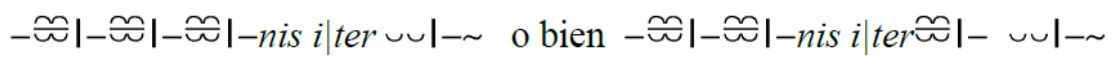

o bien

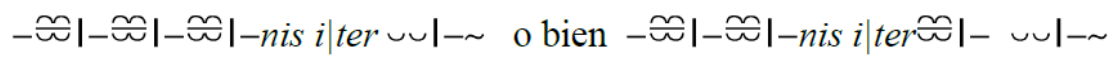

Una búsqueda en las bases de datos -EDCS y HEpOL, con carácter general, Carmina Latinae Epigraphicae Hispaniae (CLEHispaniae), para los carmina hispanos, y Musisque Deoque (MQDQ), para el conjunto de testimonios poéticos, incluidos los carmina [consultadas el día 23 de septiembre de 2018]- confirma que currunt se atestigua en dos únicos epígrafes, ambos cristianos CIL XI 275 (Ravenna) y CIL II $2 / 14,90$ (=ICERV 356; Valentia); sólo el hispano es métrico y su lectura dista de estar resuelta en este y otros puntos por causa de la rotura de la pieza (cf. Corell 1989, en especial, 66, n. 18 -de donde HEp 4, 1994, 942-; Corell 1997, esto es, en IRVT 116; Gómez Pallarés 2002, 158-161, V2 -de donde HEp 12, 2002, 537-). En cualquier caso, currunt no se encuentra documentado en posición inicial en ningún metro dactílico: entre los 54 registros censados en MQDQ prima su posición en último lugar, cerrando el verso, como ocurre en el presumible testimonio valenciano de aceptar la restitución de Corell.

50 Ambos profesores, además, están vinculados al equipo de redacción del CIL XVIII/2, volumen centrado en el material epigráfico de carácter métrico de Hispania. 


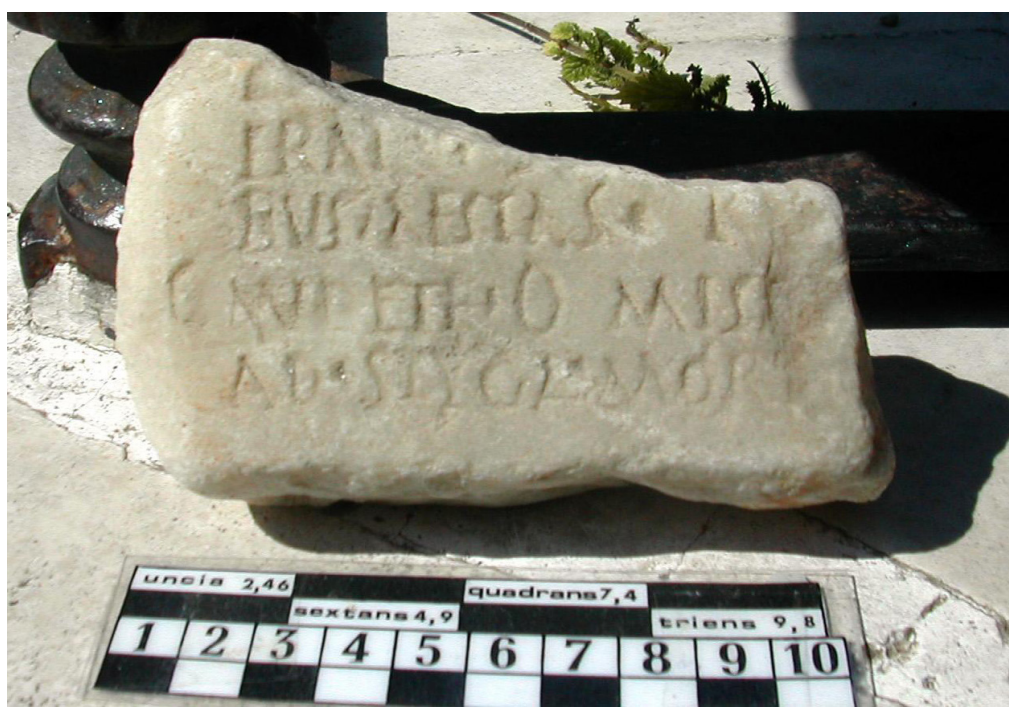

Fig. 3. Epígrafe métrico de Montemayor, $C I L \mathrm{II}^{2} / 5,506$ (C) Concepción Fernández Martínez).

Aunque ambas son posibles, indica la Dra. Carande que ninguna de las dos resulta segura: la primera porque supone una cláusula extraña, la segunda porque el verso carecería de cesura pentemímeres.

b) Líneas 2-3. Se podría proponer, en calidad de hipótesis, la siguiente restitución:

$$
\text { vivere ne du[bites tibi?] nostr }<a>\text { que temp[ora - ] }
$$

El Prof. Bolaños, autor de la restitución, apunta que en lín. 2, y para fijar la cesura, es preferible pensar en una construcción vulgar como la ofrecida -ne du[bites]- antes que considerar la presencia de $n e d u[m+$ otro monosílabo]. Señala, igualmente, que las construcciones vulgares son muy frecuentes en los CLE y que, además, la propuesta vivere ... tibi constituye una solución acorde al léxico empleado en este tipo de epígrafes.

Por lo que respecta a la lín. 3, ambos investigadores señalan que nostr $<a>$ que no resulta de su agrado: concuerdan, sí, con el hecho de que la secuencia resultante -recordemos: dos dáctilos- es métricamente correcta, pero indican, igualmente, que resulta rara y prosaica, pues constituiría una sucesión de dos palabras (=pies), lo que es infrecuente en el hexámetro.

c) Línea 4. Se propone la siguiente colometría:

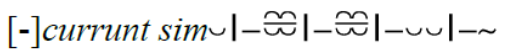

Ambos especialistas consideran que, tal y como sospechábamos, el verso no comienza en currunt, sino en una forma compuesta (occurrunt, discurrunt...), porque si-quizá de simil[-]- aparenta corresponder a una inicial breve.

Se confirmaría, en cualquier caso, que estamos ante una inscripción métrica compuesta en versos dactílicos, si bien no parece posible concretar, dada su fragmentariedad, si nos encontramos ante hexámetros en tirada o ante dísticos. 
En atención a estos datos, la restitución provisional del epígrafe podría ser:

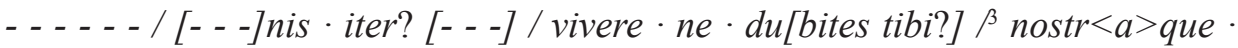
temp[ora - - - / - - ]currunt $\cdot \operatorname{sim}[---] /-\ldots$

Confirmado su carácter métrico, de proceder, como creemos, del territorio cordobés, este epígrafe-cuya datación, dados su rasgos paleográficos, encuadraríamos posiblemente en el s. II d.C.- vendría a engrosar el importante conjunto de inscripciones métricas de la provincia de Córdoba que, junto con el corpus de la de Sevilla, constituyen los mejor nutridos dentro del ámbito de la Baetica. ${ }^{51}$

\section{Conclusiones}

Lejos estábamos de saber, tras realizar el cotejo de la información contenida en la autobiografía de Castilla del Pino con las bases de datos epigráficas, que la caprichosa Fortuna nos iba a permitir conocer no dos, sino tres epígrafes inéditos presumiblemente procedentes del actual territorio cordobés. Y tres epígrafes que, cada uno a su manera, resultan de interés: el primero porque incide en la presencia y peso de los Numisii en el entorno de la antigua Colonia Patricia; el segundo porque nos proporciona el tercer registro hispano -o, mejor, cordobésde la secuencia securitati aeternae, una secuencia que, además, se encuentra escasamente atestiguada a escala imperial; el tercero, finalmente, porque constituye un nuevo documento a sumar al ya rico corpus de inscripciones métricas de la Península.

Nunca la lectura resultó tan provechosa.

\section{Referencias bibliográficas}

Abascal, J. M. (1994): Los nombres personales en las inscripciones de Hispania, Murcia.

Campos, J. M. et alii (2018): "El mundo urbano de la Bética. Breve síntesis de las ciudades de los Conventus Hispalensis y Astigitanus", [en] J. M. Campos Carrasco - J. Bermejo Meléndez (eds.), Ciudades romanas de la provincia Baetica. Corpus Vrbium Baeticarum: Conventus Hispalensis et Astigitanus. CVB I, Huelva, vol. II, 29-338.

Castilla del Pino, C. (2004): Casa del olivo. Autobiografia (1949-2003), (=Tusquets. Tiempo de memoria 42) Barcelona.

Corell, J.

(1989): "Inscripción del obispo Anesio, atribuida erróneamente a Justiniano", Saitabi 39, 63-72.

(1994): Inscripcions romanes de Saetabis i el seu territori, Valencia (=IRST).

(1997): Inscripcions romanes de Valencia i el seu territori, Valencia (=IRVT).

Fernández Martínez, C. (2007): Carmina Latina Epigraphica de la Bética romana. Las primeras piedras de nuestra poesía (=Universidad de Sevilla. Serie Lingüística 32), Sevilla $(=C L E B)$.

51 Fernández Martínez 2007, 20. 
Gómez Pallarés, J. (2002): Poesia epigràfica llatina als Països Catalans. Edició i comentari, Barcelona (=PEPC).

Grupo Mérida (2003): M. Navarro - J. L. Ramírez (coords.), Atlas antroponímico de la Lusitania romana, Mérida-Burdeos.

Haensch, R. (2016): "Safety first? CIL III, 128 et la rhétorique de la securitas", Syria 93, 2944 (http://dx.doi.org/10.4000/syria.4426).

Hübner, E. (1869): Corpus Inscriptionum Latinarum II. Inscriptiones Hispaniae Latinae, Berlin (=CIL II).

Hübner, E. (ed.), (1913): Ephemeris Epigraphica IX. Additamenta nova ad Corporis vol. II, Berlin (=EE IX).

Kajanto, I. (1965): The Latin Cognomina (=Societas Scientiarum Fennica. Commentationes Humanarum Litterarum 36/2), Helsinki.

Martín Adán, L. (2015): El latín de Hispania a partir de las inscripciones. Materiales de los conventus Astigitanus y Cordubensis, Tesis Doctoral, Universidad de Sevilla.

Martín Camacho, J. (2010): Carmina Latina Epigraphica Baeticae ex schedis: edición y comentario, Sevilla (=CLEB ES).

Melchor Gil, E. (2005): "Entre Corduba y Munda: la campaña militar del 45 a.C. y su desarrollo en la Campiña de Córdoba", [en] E. Melchor Gil - J. Mellado Rodríguez - J. F. Rodríguez Neila (eds.), Julio César y Corduba: tiempo y espacio en la campaña de Munda (49-45 a.C.), Córdoba, 363-381.

Morena López, J. A. (1998): "Reflexiones sobre el emplazamiento de Soricaria (Bellum Hispaniense XXIV y XXVII). Una nueva propuesta: El Cerro de las Cuevas de Sequeira (Castro del Río-Nueva Carteya. Córdoba)", Antiquitas 9, 31-44.

Pérez Zurita, A. D. (2017): “Sociedad cordobesa, vida municipal y mecenazgo cívico", [en] J. F. Rodríguez Neila (coord.), La ciudad y sus legados históricos. Córdoba romana (=Real Academia de Ciencias, Bellas Letras y Nobles Artes de Córdoba. Colección T. Ramírez de Arellano I), Córdoba, 121-152.

Rodríguez Neila, J. F. (2017): “Colonia Patricia (Corduba), capital de la Bética”, Gerión 35, 371-398 (http://dx.doi.org/10.5209/GERI.56152).

Ruiz Osuna, A. B.

(2007): La monumentalización de los espacios funerarios en Colonia Patricia Corduba (ss. I. a.C.-II d.C.), (=Arqueología Cordobesa 16), Córdoba.

(2009): Topografia y monumentalización funeraria en Baetica: conventus Cordubensis y Astigitanus, Córdoba.

(2010): Colonia Patricia, centro difusor de modelos. Topografía y monumentalización funeraria en Baetica (=Monografías de Arqueología 17), Córdoba.

Solin, H. - Salomies, O. (1988): Repertorium nominum gentilium et cognominum Latinarum (=ALpha-Omega 80), Hildesheim-Zürich-New York.

Stylow, A. U. (1995): “Los inicios de la epigrafía latina en la Bética. El ejemplo de la epigrafía funeraria", [en] F. Beltrán Lloris (ed.), Roma y el nacimiento de la cultura epigráfica en occidente, Zaragoza, 219-238.

Stylow, A. U. (ed.), (1995): Corpus Inscriptionum Latinarum II: Inscriptiones Hispaniae Latinae, ed. altera, pars VII. Conventus Cordubensis, Berlin-New York (=CIL II'2/7).

Stylow, A. U. et alii (eds.), (1998): Corpus Inscriptionum Latinarum II: Inscriptiones Hispaniae Latinae, ed. altera, pars $V$. Conventus Astigitanus, Berlin-New York $\left(=C I L \mathrm{II}^{2} / 5\right)$.

Vaquerizo Gil, D.

(2002): "Espacio y usos funerarios en Corduba", [en] D. Vaquerizo (ed.), Espacios y usos funerarios en el Occidente Romano. Actas del Congreso Internacional celebrado 
en el Facultad de Filosofía y Letras de la Universidad de Córdoba (5-9 de junio, 2001), Córdoba, vol. II, 143-200.

(2008): "Topografía y usos funerarios en la capital de Baetica", Archeologia Classica 59, 63-111.

(2010): Necrópolis urbanas en Baetica (=ICAC. Documenta 15), Tarragona.

(2018): "Vivir en la Córdoba romana", [en] D. Vaquerizo Gil (coord.), Los barrios en la historia de Córdoba (1). De los vici romanos a los arrabales islámicos, Córdoba, 33-116.

Vaquerizo Gil, D. - Garriguet, J. A. (coords.), (2001): Funus Cordubensium. Costumbres funerarias en la Córdoba romana, Córdoba.

Ventura Villanueva, Á.

(1999): "El teatro en el contexto urbano de Colonia Patricia (Córdoba): ambiente epigráfico, evergetas y culto imperial", AEspA 72, 57-72 (https://doi.org/10.3989/ aespa.1999.v72.296).

(2001): "Fórmulas y soportes epigráficos", [en] Vaquerizo Gil - Garriguet (coords.), 2001, 174-177.

(2002): "Nuevas inscripciones del teatro", [en] Á. Ventura-C. Márquez-A. Monterroso - M. A. Carmona (eds.), El teatro romano de Córdoba, Córdoba, 265-268.

(2009): "Las elites de Colonia Patricia, año 5 a.C.: un ejemplo de puesta en escena literaria y monumental", [en] AA.VV., Espacio, usos y formas de la epigrafia hispana en épocas antigua y tardoantigua. Homenaje al Dr. Armin U. Stylow (=AEspA, Anejo XLVIII), Madrid, 375-395. 